Methods Retrospective analysis using patients' records. 7 patients with CFRD were compared to matched CF controls using mean z-scores for weight, BMI and FEV1.

Results Records of 59 children (23 males) were analysed, 21 children between $5-10$ years and $38>10$ years. In the younger group, $80 \%(\mathrm{n}=17)$ had both $\mathrm{HbA1c}$ and random glucose tested as per our guidelines. Of 38 patients aged $>10$ years, $78 \%(\mathrm{n}=30)$ were screened by OGTT of whom $16 \%(\mathrm{n}=5)$ had the standard test. Table 1 summarises the results and shows the degree of glucose impairment on OGTT and the related grade of cystic fibrosis insulin deficiency (CFID).

Abstract P232 Table 1 The degree of cystic fibrosis insulin
deficiency (CFID) in patients ( $>10$ years) undergoing the extended
OGTT
\begin{tabular}{|l|l|l|l|}
\hline $\mathbf{N}(\%)$ & \multirow{2}{*}{ Result } & \multicolumn{2}{|c|}{ Glucose in mmol/L } \\
\cline { 3 - 5 } & & Peak & 2 hour \\
\hline $6(20)$ & Normal & - & - \\
\hline $11(36)$ & CFID1 & 28.2 & $<11.1$ \\
\hline $6(20)$ & CFID2 & $\geq 11.1$ & $<11.1$ \\
\hline $7(23)$ & CFID3 & $<7$ & $\geq 11.1$ \\
\hline $0(0)$ & CFID4 & $\geq 7$ with fasting hyperglycaemia & \\
\hline
\end{tabular}

The mean weight and BMI $\mathrm{z}$ scores for those with CFRD compared to controls were -0.64 vs $-0.02(\mathrm{p}=0.005)$ and -1.26 vs $-0.03(\mathrm{P}=0.0001)$. There was a lower trend in FEV1 in CFRD, $1.871(73.06 \%)$ vs $2.351(89.03 \%) .3$ patients with CFID3 and 1 with CFID1 later commenced insulin based on clinical grounds.

Conclusions Adherence to screening guidelines needs to be improved. Patients with CFRD have a significant declining trend in weight, BMI and FEV1 compared to controls. Some patients with CFID were commenced insulin on clinical grounds rather than results of extended OGTT. Whether treatment at earlier stages of CFID will slow down the rate of decline needs to be explored, but we have reverted back to the standard OGTT for the present.

\section{P233 COUGH SWABS SHOULD NOT BE USED TO EXCLUDE NON-TUBERCULOUS MYCOBACTERIAL (NTM) INFECTION IN ADULTS WITH CYSTIC FIBROSIS}

${ }^{1} \mathrm{C}$ Brown, ${ }^{1} \mathrm{~J}$ Choyce, ${ }^{1} \mathrm{~N}$ Rodgers, ${ }^{1} \mathrm{R}$ Rashid, ${ }^{1} \mathrm{JL}$ Whitehouse, ${ }^{2} \mathrm{EG}$ Smith, ${ }^{1} \mathrm{EF}$ Nash. ${ }^{1}$ West Midlands Adult Cystic Fibrosis Centre, Heart of England NHS Foundation Trust, Birmingham, UK; ${ }^{2}$ Health Protection Agency West Midlands Laboratory, Heart of England NHS Foundation Trust, Birmingham, UK

\subsection{6/thoraxjnl-2015-207770.369}

Introduction and objectives People with cystic fibrosis (CF) are prone to airway infection with non-tuberculous mycobacteria (NTM) including M. abscessus, M. avium and M. intracellulare. Routine NTM screening is recommended for all patients at least once a year. ${ }^{1}$ In patients not able to produce sputum, cough swabs are often sent for NTM analysis. Anecdotally, we observed that several patients cared for in our centre had cultured NTM in sputum having previously been culture-negative from cough swabs. The objective of this observational study was to examine the diagnostic yield of cough swabs and sputum samples from CF adults with known NTM infection.
Methods We identified all CF patients being cared for in our large regional adult CF centre that had cultured any NTM species from cough swabs or sputum samples and are currently attending our NTM clinic. Demographics, clinical parameters and microbiology results were recorded and analysed.

Results 26 patients (19 male) were included: median age 24 years, 92\% chronically infected with $P$. aeruginosa, 100\% pancreatic insufficient, 62\% CF-related diabetes, 27\% ABPA. 381 sputum samples and 55 cough swabs were analysed. 251 (66\%) sputum samples and $4(7 \%)$ cough swabs cultured NTM (see Table 1). In the 4 cough swabs that cultured NTM, sputum samples also cultured the same species.

Conclusions Cough swabs have a very low diagnostic yield and their use did not contribute to identification of NTM infection in our adult CF patient population. We have therefore stopped sending cough swabs for NTM culture in our centre and our data suggests that cough swabs should not be used to screen for these organisms.

\section{REFERENCE}

1 Mycobacterium abscessus Suggestions for infection prevention and control. CF Trust, 2013

\section{P234 PREVALENCE OF NON-PULMONARY COMPLICATIONS FOLLOWING LUNG TRANSPLANTATION IN ADULT PATIENTS WITH CYSTIC FIBROSIS (CF)}

S Kumar, C Etherington, P Whitaker, D Peckham. Leeds Teaching Hospitals NHS Trust, Leeds, UK

\subsection{6/thoraxjnl-2015-207770.370}

Background Lung transplantation is currently the most effective means of improving quality of life and survival in patients with end stage CF. Improvements in surgical technique, lung preservation, immune suppression and infection management have improved short and long term mortality and morbidity.

The number of significant medical complications encounter following lung transplantation can have significant impact on long term management of CF.

Aim To identify the frequency of medical complications that occur in a large cohort of post-transplant CF patients.

Method Retrospective review of electronic records to assess complications in all patients with CF who underwent Lung transplantation between September 1992 and June 2015.

Results 54 patients underwent lung transplantation (heart/lung -3 , lung/liver -1) at different transplant centres, (female-35, male-19 current median age 36 years (range,22-66), current 10, 15 and 20 year survival rates are 43\%, 22\% and 7\% respectively (median 87 months). Complications are shown in Table 1.

$9(17 \%)$ post-transplant patients died (median survival 77 months). Of these, 3 (33\%) died secondary to malignancy.

Conclusion In addition to organ rejection and infective causes common systemic complications included hypertensive disease (50\%), gastro-oesophageal reflux disease (30\%), chronic kidney disease (26\%), and osteoporosis (19\%). It is notable that one third of patient mortality was due to malignancy. As patient survival improves we may need to consider increased screening of these high risk patients. Frequent monitoring and excellent collaboration between transplant and CF centres may lead to earlier detection and treatment of these complications. 


\begin{tabular}{lll}
$\begin{array}{l}\text { Abstract P234 Table 1 } \\
\text { lung transplantation }\end{array}$ & Non pulmonary complications following \\
\hline GIT: & Cardiovascular: & Malignant Disease: \\
$\begin{array}{l}\text { Gastro-oesophageal Reflux } \\
\text { disease (GORD) (16) }\end{array}$ & Hypertensive disease & $\begin{array}{l}\text { Post-transplant } \\
\text { Nissen fundoplication (12) }\end{array}$ \\
$\begin{array}{l}\text { Bowel obstruction (4) } \\
\text { Oesophageal candidiasis (3) }\end{array}$ & Myocardial infarction & (2) \\
Oesophageal fistula (1) & Pericarditis (1) & Squamous and basal cell \\
Gastrointestinal bleed (1) & & carcinoma- Skin (3) \\
Duodenitis (1) & & Cervical- Squamous cell \\
& & carcinoma (1) \\
& & Gastric adenocarcinoma (1) \\
Renal: & Mulvar intraepithelial neoplasis \\
& complication: & (1) \\
Chronic kidney disease (CKD) (14) & Osteoporosis (10) & Steroid related Diabetes (2) \\
(requiring renal transplantation-3, & Osteopenia (10) & Adrenal insufficiency (1) \\
haemodialysis - 1) & Gouty arthropathy (2) & Secondary hyper- \\
Acute Kidney injury (3) & Vertebral compression & parathyroidism (1) \\
& fracture (1) & \\
\hline & &
\end{tabular}

P235 TEMOCILLIN FOR BURKHOLDERIA INFECTION IN CYSTIC FIBROSIS - ADDING TO TREATMENT OPTIONS?

S Ajab, J Marlow, K Delisle, M Walshaw, M Ledson. Liverpool Heart and Chest Hospital, Liverpool, UK

\subsection{6/thoraxjnl-2015-207770.371}

Introduction Burkholderia spp infection in cystic fibrosis (CF) confers a worse prognosis, with increased morbidity and mortality. Treatment can be problematic because strains are often resistant to many of the commonly available intravenous antibiotics, leading to a search for new therapies. We have studied the use of Temocillin, a derivative of Ticarcillin with promising in-vitro activitiy against Burkholderia spp, ${ }^{1}$ in the treatment of acute exacerbations in CF patients infected with Burkholderia spp.

Methods We have used Temocillin in IV form, often in combination, in acute respiratory exacerbations in CF patients attending our large adult unit who are chronically infected with Burkholderia spp. We present our two year data, looking at the subspecies treated, length of treatment, improvement in clinical parameters, and co-antibiotic use.

Results Nineteen courses of IV Temocillin were administered to 7 CF patients (mean age 29 years, [range 23-42 years], 2 males, 1 infected with B Cenocepacia, 6 with B Multivorans.) All patients completed their treatment without complication.

Median length of Temocillin treatment was 9.7 days (range 3-16 days), and most patients finished their therapy as outpatients (mean inpatient stay 5.2 days). All patients had clinical improvement, with all gaining weight (mean $3 \mathrm{~kg}$ [range 6.5 to $0.9]$ ) and most increased spirometry (mean change in FEV1\% predicted $6[17$ to -3$])$.

As regards the efficacy of co-antibiotic use, the 11 courses accompanied by Ceftazidime had a mean $3 \%$ improvement in FEV1, 8\% with Meropenem (6 courses) and 9\% with Colomycin ( 2 courses).

Discussion This clinical study has shown that Temocillin is well tolerated by $\mathrm{CF}$ patients and is associated with clinical improvement in those infected with Burkholderia spp when given in combination with other antibiotics. Temocillin adds to the limited antibiotic armamentarium available to treat these difficult infections in CF patients and we recommend its use to other clinicians.

\section{REFERENCE}

1 Bonacorsi S, Fitoussi F, Lhopital S, Bingen E. Comparative in vitro activities of meropenem, imipenem, temocillin, piperacillin, and ceftazidime in combination with tobramycin, rifampin or ciprofloxacin against Burkholderia cepacia isolates from patients with cystic fibrosis. Antimicrob Agents Chemother. 1999;43: 213-217

\section{Clinical studies in cough}

\section{P236 PSYCHOLOGICAL PROFILE OF INDIVIDUALS PRESENTING WITH CHRONIC COUGH}

${ }^{1} \mathrm{~K}$ Hulme, ${ }^{1} \mathrm{~S}$ Dogan, ${ }^{2} \mathrm{~V}$ Deary, ${ }^{1} \mathrm{SM}$ Parker. ${ }^{1}$ Respiratory Medicine, North Tyneside Hospital, Northumbria Healthcare NHS Foundation Trust, North Shields, UK; ${ }^{2}$ Northumbria University, Newcastle, UK

\subsection{6/thoraxjnl-2015-207770.372}

Introduction and objectives Between $20-40 \%$ of patients seen in respiratory clinics with chronic cough have an 'idiopathic cough' with persistent symptoms that are refractory to treatment and have no obvious underlying pathology. Adverse consequences of chronic cough are well documented in the literature, but relatively little is known about this patient population. We aimed to investigate the association of psychological factors, identified as important in the medically unexplained, persistent symptom literature, with chronic cough.

Methods Eighty-nine participants (63 female, mean age $=59$ ) took part. Sixty-seven patients attending a specialist cough clinic (idiopathic; $\mathrm{n}=25$, explained cough; $\mathrm{n}=42$ ) and 22 normal controls were asked to complete questionnaires; all participants completed the Hospital Anxiety and Depression Scale, Big Five Inventory (Personality), Chalder Fatigue Scale and Patient Health Questionnaire-15. Cough patients also completed the Illness Perception Questionaire-Revised. Appropriate statistical analyses were conducted comparing the participant groups.

Results Idiopathic coughers displayed significantly higher levels of neuroticism $(p<0.05)$, anxiety $(p<0.05)$, depression $(p<$ $0.001)$, fatigue $(\mathrm{p}<0.001)$ and somatic physical symptoms $(\mathrm{p}<$ $0.005)$ than controls. In comparison to explained coughers, significantly higher depression $(\mathrm{p}<0.005)$ and fatigue $(\mathrm{p}=0.01)$ scores were reported by idiopathic coughers, who also had significantly more negative illness perceptions $(p<0.005)$. Specifically, they had strong beliefs regarding negative consequences, lower illness coherence and higher emotional representations. Explained coughers only differed significantly to the control group in the increased levels of fatigue reported $(p<0.05)$.

Conclusions Many psychological factors are associated with chronic cough and seem to differentiate between the two patient groups. The prevalence of neuroticism, negative affect, negative illness beliefs and increased physical symptom reporting suggest a patient profile of idiopathic cough similar to that of other medically unexplained symptoms. This, as well as the novel and significant finding of the prevalence of fatigue, should be considered in consultations and developing novel interventions. 